Carta a o editor / Letter to editor

\section{Leucemia Mielóide Aguda t(8;21) após tratamento de hipertiroidismo com iodo radioativo: Leucemia secundária?}

\author{
Daniella M.M. Bahia ${ }^{1}$ \\ Maria L.F. Chauffaille \\ Maura S. Romeo ${ }^{1}$ \\ Magnus R. Dias-Silva ${ }^{2}$ \\ José Kerbauy ${ }^{1}$
}

1 - Disciplina de Hematologia e Hemoterapia. Escola Paulista de Medicina/UNIFESP

2 - Disciplina de Endocrinologia. Escola Paulista de Medicina/UNIFESP

\section{Sr. Editor,}

Paciente do sexo feminino de 33 anos foi diagnosticada com hipertiroidismo assintomático em setembro de 1992 (TSH <0,05; T4L=1,6) e em maio 1993 recebeu $10 \mathrm{mCi}$ de lodo ${ }^{131}(370 \mathrm{MBq})$ com normalização da função tireoidiana. Três anos após, apresentou pancitopenia e o mielograma revelou LMA-M2, de acordo com classificação morfológica $\mathrm{FAB}$ e a citogenética apresentou $46, \mathrm{XX}, \mathrm{t}(8 ; 21)$ ( $\mathrm{q}$ 22; q22) [20]. Foi iniciada quimioterapia com citosina arabinosídeo e daunorrubicina, evoluindo a óbito por sepsis durante o período de granulocitopenia pós terapia de indução.
A leucemia Mielóide Aguda (LMA) após terapia com agentes alquilantes, radioterapia e inibidores da topoisomerase II é uma condição bem reconhecida. LMA pós tratamento com agentes alquilantes e radioterapia geralmente apresenta uma fase mielodisplásica antes de evoluir para leucemia e tem como achados citogenéticos característicos a deleção ou perda do cromossomo 5 ou 7. Enquanto a LMA relacionada ao uso de epipodofilotoxinas, inibidores da topoisomerase II, já se apresenta como franca LMA, sem história prévia de mielodisplasia, com translocações balanceadas envolvendo os cromossomos 11q23 e 21q22 (1).

Leucemia pós-tratamento com lodo radioativo é um fato raro, com poucos relatos na literatura e incidência em torno de $2 \%$ em pacientes com câncer de tireóide. Em hipertiroidismo, os dados são mais conflitantes, tempo e dose de exposição ao lodo são fatores importantes na leucemogênese $(2,3,4)$. Hall e Holm estudando 47.712 pacientes avaliaram o risco de desenvolver câncer pós exposição a lodo ${ }^{131}$ para fins diagnósticos, tratamento de câncer de tireóide e hipertiroidismo, não demonstrando uma associação convincente entre 10 do ${ }^{131}$ e risco aumentado de câncer ou leucemia, à exceção do câncer de estômago. Outras neoplasias hematológicas têm sido relatadas após exposição a lodo ${ }^{131}$, como leucemia linfóide crônica e leucemia mielóide crônica (LMC). A LMC é descrita após exposição a diferentes doses de iodo e com intervalo de tempo entre exposição e diagnóstico

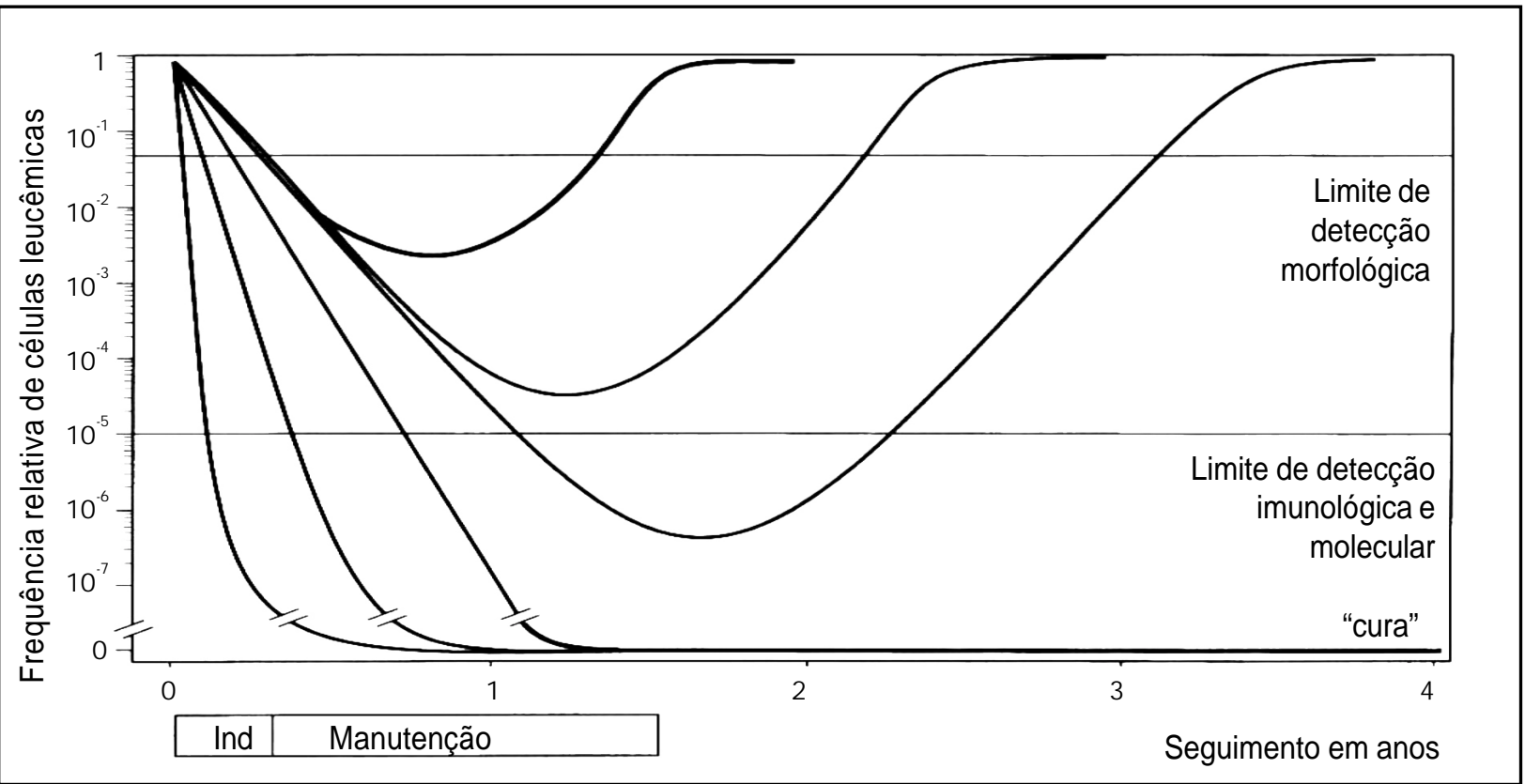


variável, mas ainda assim nem todos os casos foram confirmados com o achado do cromossomo Philadelphia, sendo, portanto, este diagnóstico questionável.(5)

A LMA secundária com $t(8 ; 21)$ (q22; $q 22)$ é um evento raro e se caracteriza por um curto período de latência, sem sinais de mielodisplasia ou relato de tratamento prévio com agentes inibidores da topoisomerase II e radioterapia (6).

Relatamos um caso de LMA com $\mathrm{t}(8 ; 21)(q 22 ; q 22)$ após tratamento com lodo ${ }^{131}$ para hipertiroidismo, cuja apresentação clínica foi semelhante às demais LMAs secundárias com $t(8 ; 21)$. Embora o potencial carcinogênico do lodo ${ }^{131}$ ainda seja questionável, a intenção é ressaltar aqui que esta associação pode não ser ao acaso, uma vez que Bloomfield et al também demonstraram um caso de LMA-M 2 pós tratamento com lodo ${ }^{131}$ para hipertiroidismo que, no entanto, apresentava cariótipo normal.(7)

Por outro lado, não podemos deixar de considerar que estes eventos podem não estar diretamente relacionados e esta LMA $\mathrm{t}(8 ; 21)$ ser uma leucemia de novo, reafirmando a dificuldade em estabelecer relação entre estes tipos de leucemias e seus prováveis agentes causais. Diante disso não podemos deixar de relatar este caso, já que mais estudos são necessários para elucidar esta questão.

\section{AcuteM yeloid Leukemiat(8;21) after treatment for hyperthyroidism using radioactiveiodine: Secondary Leukemia?}

Daniella M.M. Bahia, Maria L.F. Chauffaille, Maura S. Romeo, Magnus R. Dias-Silva, José Kerbauy

\section{Abstract}

Acute leukemia following treatment with lodine131 is a rare event. The possible carcinogen ic effect of lodine131 is still not clear and a large series of cases did not show an increased incidence of cancer. A case of AML $t(8 ; 21)$, three years after Iodine131 treatment for hyperthyroidism, is reported.

Secondary AML with $t(8 ; 21)$ is described following exposure to drugs that target topoisomerase II and radiotherapy. The controversial potential of lodine131 as a leukemogenic agent and the fact that $t(8 ; 21)$ is also found in de-novo AML, emphasize the problem in establishing a relationship between these events although this potential can not be ruled out.

\section{Referências Bibliográficas}

1 .Pedersen-Bjergaard J, Pedersen M, Roulston D, Philip P. Different genetic pathways in leukemogenesis for patients presenting with therapy-related myelodysplasia and therapyrelated acute myeloid leukemia. Blood, 1995; 86(9):3542-52.

2. Hall $P$, Holm LE. Cancer in iodine-131 exposed patients. J Endocrinol Invest 1992; 18: 147-9.

3. Laurenti L, Salutari P, Sica S, Piccirillo N, Zini G, Zollino M, Leone G. AcuteMyeloid Leukemia after iodine-131 treatment for thyroid disorders. Ann H ematol, 1998; 176:271.

4. Bitton R, Sachmechi I, Benegalrao Y, Schneider BS. Leukemia after a small dose of radiodine for metastatic thyroid cancer. J Clin Endocrinol M etabol 1993; 77(5):1423-26.

5. Shimon I, Kneller A, Olchovsky D. Chronic myeloid leukemia following ${ }^{131}$ I treatment for thyroid carcinoma: a report of two cases and review of the literature. Clin Endocinol, 1995; 43:651-4.

6. Leseve JF, Schneider P, Dolgopolov I, Bastard C, Lenormand $B$, Cambon-Michot $E$, Callat MP, Caveleier B, Tron PH, Vannier JP. Therapy-related acute myeloid leukemia with $t(8 ; 21)$ in a child with previousEwing's Sarcoma. M ed Ped O ncol 1997; 29:132-4.

7. Bloomfield CD, Shuma C, Regal L, Philip PP, Hosfeld DK, Hagemeijer AM, Garson OM, Peterson BA, Sakurai M, Alimena G, Berger R, Rowley JD, Ruutu T, Mitelman F, Dewald GW, Swansbury J. Long term survival of patients with acute myeloid leukemia: a third follow-up of the forth international workshop on chromosomes in leukemia. Cancer 1997; 80(11):2191-8.

$$
\begin{aligned}
& \text { Recebido - 07/03/2002 } \\
& \text { Aceito - 17/06/2002 }
\end{aligned}
$$

\footnotetext{
Correspondência para: Maria de Lourdes F.

Chauffaille

Disciplina de Hematologia e Hemoterapia

Escola Paulista de Medicina/UNIFESP

Rua Botucatu, 740

CEP: 04023-900. São Paulo. SP

Fone: (11) 5579-1550/ 5576-4240

Fax: (11) 5571-8806

E-mail: chauffaill@hemato.epm.br
} 